\title{
Green Synthesis of Copper nanoparticles using Zingiber officinale Extract and Characterization
}

\author{
Hari Bhakta Oli ${ }^{1 *}$, Nabin Sharma ${ }^{2}$, Ekaraj KC ${ }^{2}$, Anup Subedee ${ }^{1}$ and Rajan Timilsina ${ }^{3}$ \\ ${ }^{1}$ Amrit Campus, Tribhuvan University, Kathmandu \\ ${ }^{2}$ Mahendra Multiple Campus, Tribhuvan University, Dang \\ ${ }^{3}$ Melamchi Water Supply Development Board, Kathmandu, Nepal \\ Email: haribhakta69@gmail.com
}

\begin{abstract}
Copper nanoparticles were synthesized in laboratory by green method in much efficient way. Extract from Zingiber officinale was prepared in laboratory and used to reduce copper(II) form its salt to copper nanoparticles. The extract contains various alkaloids, flavonoids and phenolic groups which acts as reducing as well as capping agents. CuNPs are obtained by stirring 0.01M copper(II) sulphate solution with extract in the ratio 5:3 at moderate temperature $\left(<60^{\circ} \mathrm{C}\right)$. The formations of nanoparticles were detected by UV spectrophotometer. The maximum absorbance at 595nm indicates the formation of nanoparticle. Presence of reducing and stabilizing agent i.e. conjugate double bond and different functional groups of, flavonoids and polyphenolic group in extract of Zingiber officinale was also detected by UV spectrophotometer in the range of 295, 310, 350 and $375 \mathrm{~nm}$. Those nanoparticles were characterized by using EDX and FTIR. Characterization by using XRD shows CuNPs possesses face centered cubic crystal lattice structure and average size of CuNPs is $31 \mathrm{~nm}$.
\end{abstract}

Key words: CuNPS, EDX, extract, green method, XRD

\section{Introduction}

Materials which have at least one dimension (length or width or height) in the range of $1-100 \mathrm{~nm}$ are called nanomaterial. Nanomaterials have high application potentials due to their unique mechanical, thermal, electrical, magnetic, catalytic and optical properties. Generally, metal and metal oxide nanoparticles have great use in our daily life ${ }^{1}$. Nanoparticles (NPs) show size and shape dependent properties so they are totally different from bulk materials. Large surface area of NPs gives unique characteristics such as optical properties and Surface Plasmon Resonance ${ }^{1,2}$.

NPs are of great interest due to their small size and high surface to volume ratio that leads to chemical and physical differences in their properties such as mechanical properties, biological and steric properties, catalytic activity, electrical and thermal conductivity, optical absorption and melting point compared to that of bulk materials ${ }^{1,3}$. Recently many metal NPs like copper, silver, gold, magnesium, zinc, titanium have been taken as hot topic for research because of their antimicrobial activity against different bacteria ${ }^{4}$.

\footnotetext{
* Corresponding author
} 
Hence, they are extensively applied in medicine, dental materials, water treatment, sunscreen lotions and coatings etc. ${ }^{4,5}$. Not only metal nanoparticles but also metal oxides, ceramics, silicates and polymer nanoparticles have been synthesized and employed in variety of applications like catalysis, sensing and optoelectronics $^{6,7}$.

Due to increased demand for nanoparticles over the past few decades, a wide range of physical and chemical techniques have been developed to synthesize NPs of different sizes, shapes and compositions. The physical approach to synthesize NPs include laser ablation, lithography and high energy irradiation ${ }^{9}$ while the chemical approach includes chemical reduction, electrochemistry and photochemical reduction ${ }^{4,7,10}$. Studies have shown that during the synthesis process; size, shape, stability and physicochemical properties of the NPs are strongly influenced by variety of factors. These factors include process parameters (temperature, concentration) process kinetics and adsorption kinetics involving the stabilizing agent ${ }^{11,12}$.

Synthesis of copper and copper-based nanoparticles is limited compared to gold and silver nanoparticles. The main difficulty lies in their preparation and preservation as they oxidized immediately when exposed in air. Scientists are using different inert media such as Argon, Nitrogen to overcome this oxidation problem also using reducing and capping or protecting agents for the reduction of copper salt used. Polymers are also used to stabilize the synthesized copper nanoparticles ${ }^{4,15,17}$. Copper nanoparticles (CuNPs) are generally synthesized by hydrothermal method, microwave assisted pylol method, thermal reduction etc. However, these methods are costly and utilize harsh organic solvents ${ }^{2,17}$. Consequently designing a process that effectively controls the size, shape, stability and physicochemical properties is currently at the forefront of research into NPs synthesis. Conventional synthesis of NPs can involve expensive chemical and physical processes that often use toxic materials with potential hazards such as environmental toxicity, cytotoxicity and carcinogenicity ${ }^{10}$ the toxicity problem arise from the hazardous substances such as organic solvents, reducing agents and stabilizers that are used to prevent unwanted agglomeration of colloids. Because of the presence of these toxic materials, the NPs have been prevented from their clinical and biomedical applications. Importantly, all these factors can be potentially controlled via biological mediated production. As the result, there is currently widespread interest in developing clean, reliable, biologically compatible, benign and environment friendly green processes to synthesize $\mathrm{NPs}^{13,14}$.

Synthesizing nanoparticles via biological entities offers clean, nontoxic and environment friendly method. It is the great value for synthesizing well known nanoparticles from ancient times for its medicinal values ${ }^{13}$. Many bacteria, fungi and plants have shown the ability to synthesize metallic NPs. Intracellular or extracellular synthesis, growth temperature, synthesis time and ease of extraction, all play important role in biological NPs production ${ }^{18}$ in biological method, the plant extract are used as reducing agent and capping agent for the synthesis of NPs due to the presence of biomolecules with antioxidant or reducing properties ${ }^{11,16}$.

Green methods of synthesizing nanoparticles using Plants, microorganisms and enzymes have several advantages over conventional physical and chemical methods. The synthesis of CuNPs is a very challenging task because they have relatively lower redox potential, and it is prone to oxidation when it is 


\section{J. Nepal Chem. Soc., Vol. 39, 2018}

exposed to air. Interest in synthesis of copper nanoparticles arises from the useful properties of this metal such as the good thermal and electrical conductivity and a cost much less than silver. This leads to potential application in cooling fluids for electronic systems, in the field of sensors, catalysis and inkjets $^{6,8,18}$. The green synthesis is best alternate for the synthesis of nanoparticle among other method due to its cost effectiveness, easily available materials, environment friendly and of high \% yield of nanoparticles. Metal nanoparticles have been used in medicine and agricultural field ${ }^{8,18}$.

Zingiber officinale is medicinally important herb. In this research, Zingiber extract is used for the synthesis and stabilization of CuNPs. Since the extract contains alkaloids, flavonoids and phenolic groups which potentially can act as reducing agents. These compounds also act as capping agent so no any other capping agents are required. As a result of the abundant and desirable medicinal characteristics of Zingiber, NPs synthesized from its extract may be applicable in biomedical therapies, diagnosis and sensing ${ }^{19,20}$. The application of nanoparticles in different propose is increasing day by day. But the method of preparation for nanoparticle needs high cost and less yields. So, in this work environment friendly, less chemical hazardous and for better yield with low cost, green synthesis is selected.

\section{Experimental Methods}

\section{Preparation of plant extract}

Rhizomes of Zingiber officinale plant were collected from Kalimati Kathmandu. These rhizomes were crushed and grinded by using mortar and pestle. Now, $300 \mathrm{~g}$ of paste was taken and mixed with $1 \mathrm{~L}$ of distilled water and stirred vigorously. Such diluted paste was filtered by using Whatmann no. 40 filter paper. Filtrate was taken as extract of plant.

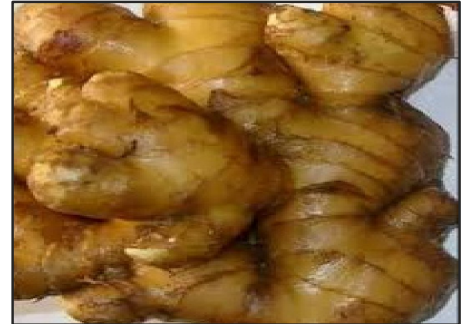

Figure 1: Rhizome of Z. officinale

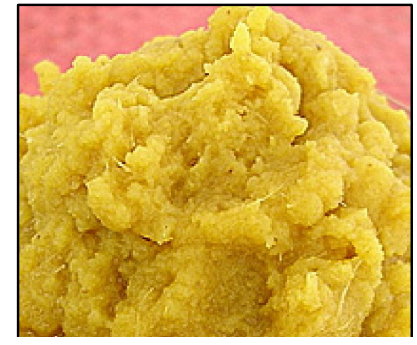

Figure 2: Paste of Z. officinale

\section{Synthesis of Nano particles}

CuNPs were synthesized by using Zingiber officinale extract. For this process stock solution of copper sulphate and plant extract were mixed in different ratio and stirred in magnetic stirrer up to 6 hours. The good yield was obtained when copper sulphate and plant extract were mixed in (5:3) ratio at 4 hrs. i.e. 50 $\mathrm{mL}$ of copper sulphate solution was mixed with $30 \mathrm{~mL}$ of Zingiber extract. Temperature of mixture is maintained below $60{ }^{\circ} \mathrm{C}$ in order to control the evaporation of volatile organic compounds from extract of plant. Actually, $\mathrm{pH}$ of the solution also effects on the formation of nanoparticles. The $\mathrm{pH}$ of the solution must be acidic. Here, we maintained $\mathrm{pH}$ of solution below 5 without addition of additives. Within 4 hours, the blue color solution was change into brown color, which indicates the formation of CuNPs. Then the solution was centrifuged for $20 \mathrm{~min}$ at $8000 \mathrm{rpm}$ and dispersed in distilled water to remove any unwanted biological materials. 


\section{Characterization of nanoparticles}

In visual characterization, the bio reduction of copper sulphate using aqueous Zingiber officinale extract was monitored by observing the color change after mixing. The appearance of brown color indicates the formation of copper nanoparticle.

The reduction of copper sulphate to copper nanoparticles(CuNPs) was monitored by measuring UVVisible spectrum of the reaction medium over the range $400-700 \mathrm{~nm}$ at interval of $1 \mathrm{~nm}$. The measurements were recorded in every $1 \mathrm{hrs}$ interval by taking a small aliquot of the sample in cuvette and it was also diluted according to its measurement.

Similarly, Fourier transformed infrared (FTIR) spectroscopic study was carried out to ensure that there are strong functional groups in extract. For FTIR measurements, a drop of pure sample was used to record peaks which were done in attenuated total reflectance (ATR) mode. The data were recorded in the wavelength range from $4000 \mathrm{~cm}^{-1}$ to $750 \mathrm{~cm}^{-1}$ at the resolution of $8 \mathrm{~cm}^{-1}$.

The crystal structure of synthesized CuNPs was studied with the help of X-ray diffraction (XRD). Diffraction pattern was obtained for CuNPs powder synthesized by the green method, using Rigaku Multiflex diffractometer with monochromated $\mathrm{CuK \alpha}$ radiation of the wavelength $1.5406 \AA$. XRD scanning was performed under ambient conditions over the $2 \theta$ region of $10^{\circ}-80^{\circ}$ at the rate of $2^{\circ} / \mathrm{min}$ $(40 \mathrm{kV}, 20 \mathrm{~mA})$ in Nepal Academy of Science and Technology (NAST) Khumaltar, Lalitpur, Nepal. The crystalline domain size of synthesized CuNPs was calculated using Debye-Scherrer's formula.

$$
\begin{aligned}
& \mathrm{D}=\frac{0.9 \lambda}{\beta \cos \theta} \ldots \ldots \ldots \text { (1) } \\
& \text { Where, } \\
& \mathrm{D}=\text { grain size }=\text { diameter of nanoparticle } \\
& \lambda=\text { wavelength of } \mathrm{X} \text {-ray used } \\
& \beta=\text { full width at half maximum intensity (in radian) of intensity profile } \\
& \theta=\text { diffraction angle }
\end{aligned}
$$

\section{Results and Discussion}

Results of green synthesis of copper nanoparticles by reduction of aqueous copper(II) ions using Zingiber officinale extract and the progress of formation of CuNPs was monitored by color change, UVVis spectroscopy, EDX, FTIR and XRD.

When CuNPs were synthesized using Zingiber officinale extract as reducing and stabilizing agent, the blue color of copper sulphate pentahydrate solution was turned to brown color indicating the formation of $\mathrm{CuNPs}^{21}$ which is shown in figure 3.

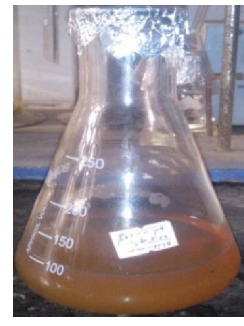

Figure 3: Brown colored solution after formation of CuNPs 


\section{J. Nepal Chem. Soc., Vol. 39, 2018}

When copper sulphate solution was mixed with yellowish extract of Zingiber officinale the color of mixture immediately changes into light green which indicates the reduction of copper ion. The color of solution gradually changes with increasing time of stirring and finally brown colored solution were obtained. Therefore, Zingiber extract completely reduced $0.01 \mathrm{M} \mathrm{CuSO}_{4}$ solution with in 4 hrs to form CuNPs.

The UV-Visible spectra of pure plant extract (Zingiber officinale juice) shows absorption peak at 295, 315,355 and $375 \mathrm{~nm}$ is due to presence of aromatic compound, carboxylic group, phenolic group and substituted phenolic group as shown in figure 4.

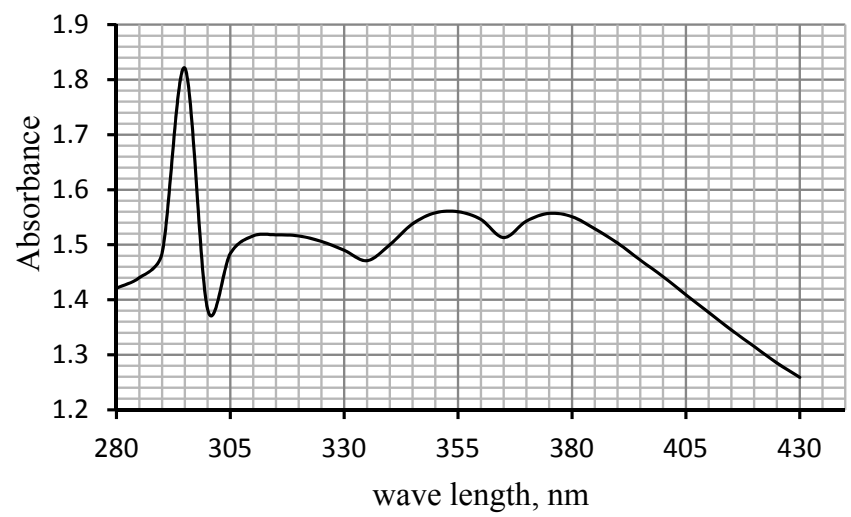

Figure 4: UV spectra of plant extract.

Copper sulphate solution and extract was mixed in different ratio and absorbance was measured. The absorbance peak on UV-Visible spectrophotometer for (3:5) ratio of extract and precursor solution up to 4 hrs is given in figure 5. This mixture gives good result for synthesis of nanoparticle at $4 \mathrm{hrs}$.

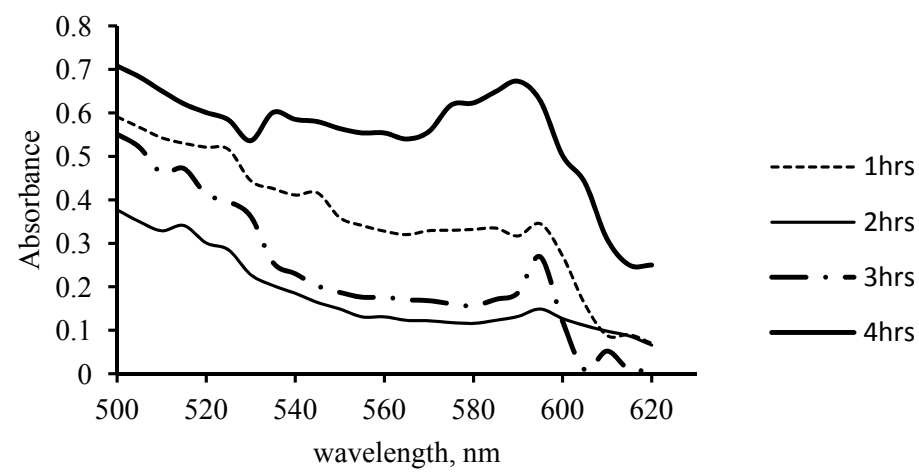

Figure 5: UV-Visible absorbance peak of mixture(3:5) in different interval of time 
The reduction of $\mathrm{Cu}^{2+}$ ions to CuNPs using extract was indicated by the change of color from light green to brown. It may be due to Surface Plasmon Resonance (SPR) effect and reduction of copper ion. Then the characteristic absorption peak gradually increases with time as shown in above figure 5, 6 and 7. The maximum absorption peak of CuNPs appeared at $596 \mathrm{~nm}$ after $4 \mathrm{hrs}$ which indicates the formation of $\mathrm{CuNPs}^{17}$. The synthesized and stabilized CuNPs is further conformed by EDX.

Energy dispersive X-ray (EDX) studied was carried out to know about the elemental composition of the sample. The elemental profile of copper has been conformed for biosynthesized CuNPs using plant extract. The elemental analysis of nanoparticle yielded $67.808 \%$ copper which proves that produced CuNPs were in highest purified form with small impurities. The peak at $8 \mathrm{KeV}$ is due to CuNPs. Elements present in CuNPs synthesized from plant extract are tabulated as in table 1.

Table 1: Elemental composition in synthesized CuNPs by EDX method

\begin{tabular}{|l|l|}
\hline Elements & Weight \% \\
\hline $\mathbf{C u}$ & 67.808 \\
\hline $\mathbf{S}$ & 12.928 \\
\hline $\mathbf{S i}$ & 17.944 \\
\hline $\mathbf{K}$ & 1.073 \\
\hline $\mathbf{Z n}$ & 0.248 \\
\hline & \\
\hline
\end{tabular}

X-ray diffraction (XRD) pattern of synthesized CUNPs is shown in figure 6. The result of XRD shows that the synthesized CuNPs are crystalline in nature as attested by clearly defined sharp peaks. The diffraction peaks were obtained at $2 \theta$ angles of $43.56^{\circ}, 50.50^{\circ}$, and at $74.09^{\circ}$, which can be indexed to (111), (200), and (220) lattice planes respectively of $\mathrm{Cu}$ crystals. XRD pattern obtained further shows that the synthesized CuNPs possess face centered cubic (FCC) lattice structure.

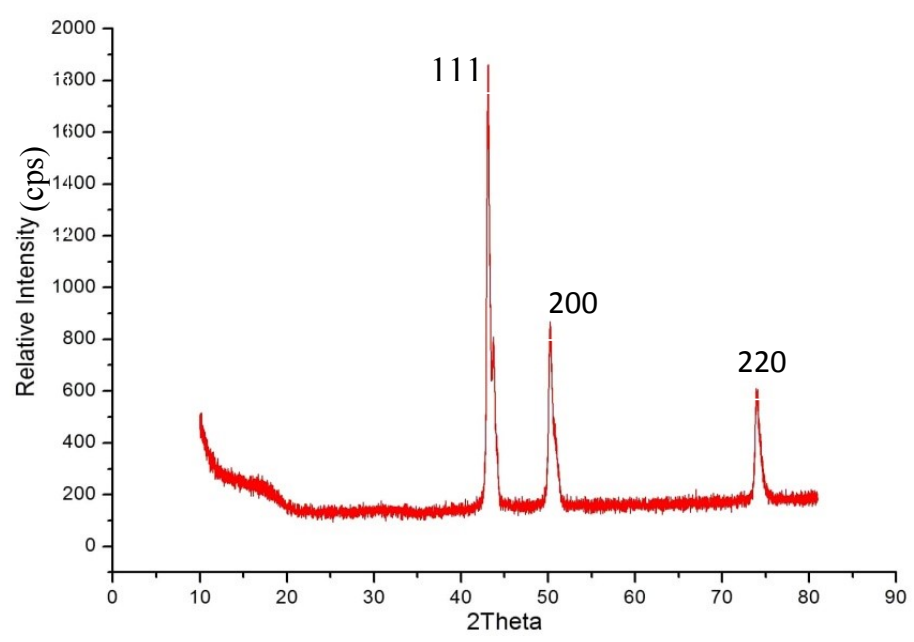

Figure 6: X-ray diffraction pattern of CuNPs 


\section{J. Nepal Chem. Soc., Vol. 39, 2018}

The XRD patterns obtained do not contain any other noticeable peaks corresponding to any other copper related compounds suggests that the synthesized CuNPs are free from oxidation. However, the tiny peak as branch on (111) plane shows that CuNPs contains some impurities. The nano crystallite size (D) was determined by using Debye- Scherrer's formula:

$$
\mathrm{D}=\frac{0.9 \lambda}{\beta \cos \theta}
$$

Where, $\lambda$ is the $\mathrm{X}$ - ray wavelength ( $\lambda=1.54056 \AA$ for $\mathrm{Cu} \mathrm{K} \alpha$ radiation), $\theta$, Braggs diffraction angle, $\beta$ is the full width half maximum (FWHM) in the radians of the XRD peak at the diffraction angle $\theta$. A constant 0.9 appears as a shape factor for most of the spherical crystals. Based on XRD result, one can conclude the formation of well capped highly crystalline pave CuNPs. Substituting the corresponding values on Scherrer's equation, the crystalline size for (111) peak of CuNPs was determined by using above equation. As the given value for (111) plane crystalline size are $\mathrm{K}=0.9, \lambda=1.54056 \AA, \beta=1.0116$ $\times 10^{-2}$, and $\theta=43.56^{\circ}$, the approximate particle diameter was calculated and found to be $31 \mathrm{~nm}$. The size of synthesized CuNPs reported in literature was in the range of $35-75 \mathrm{~nm}^{22}$.

\section{Conclusions}

CuNPs were successfully synthesized by reducing copper sulphate with plant extract (Zingiber officinale juice). The green synthesis of CuNPs is simple, cost effective and ecofriendly. CuNPs are characterized by UV-Visible spectroscopy, FTIR analysis and EDX analysis. Results of UV-Visible spectrum showed maximum absorption peak at 596nm. Similarly, FTIR suggested that role of alcoholic/phenolic group as well carbonyl group containing organic compounds to form CuNPs as well as stabilization. Likewise EDX spectrum further supported for the presence of high percentage of copper along with some other impurities. The highly intense peak at $8 \mathrm{eV}$ in EDX spectrum is due to presence of CuNPs. Similarly, XRD analysis shows that the CuNPs possesses face centered cubic lattice structure and the size of CuNPs is $31 \mathrm{~nm}$.

\section{Acknowledgements}

Authors are grateful to the Customs Department for EDX and FTIR analysis, Nepal Academy of Science and Technology, Khumaltar, Lalitpur, for XRD analysis, Amrit Campus for laboratory facility and IOST, Office of Dean, TU, Kathmandu for financial support.

\section{References}

1. B.B. Neupane, B. Pandey, B. Giri and M.K. Joshi, A text book of nanoscience and nanotechnology, published by Heritage publishers and distributers Pvt. Ltd., 2016, ISBN:9789937-595-87-2

2. S. Shaik, L. Mkize, M. Khumalo and N. Singh, Afr. J. Tradit Complement Altern. Med., 2015, 12, 33-38.

3. S. Iravani, Green chemistry, 2011, 13, 2638-2650.

4. M. Imran Din, and R. Rehan, Analytical letters, 2016, doi: 10.1080/00032719.2016.1172081.

5. R. Mittu, Int. Adv. Res. J. in Sci., Eng. and Tech., 2016, 3, 37-40. 
6. T.M. Dung Dang, T.T. Thu Le, E. Fribourg-Blanc, and M.C. Dang, Adv. Nat. Sci.: Nanosci. Nanotechnol. 2011, 2, doi:10.1088/2043-6262/2/1/015009.

7. A. Eman and A.A. Rasha, Int. J. of Electrochem. Sci., 2016, 11, 4712-4723.

8. V.N. Kalpana, P. Chakraborthy, V. Palanichamy and V. D. Rajeswari, Int. J. of Chem. Tech. Research, 2016, 9(09), 498-507.

9. A.D. Karthik, and K. Geetha, J. of Applied Pharmaceut. Sci., 2013, 3(5), 16-21.

10. P.C. Nagajyothi, P. Muthu Raman, T.V.M. Sreekantha, D.W. Kim and J. Shim, Arab. J. of Chem., 2016, doi: 10.1007/s10876-016-1082-z

11. G. Caroling, M.N. Priyadharshaini, E. Vinodhini, A.M. Ranjitham and P. Shanthi, Int. J. of Pharm. And Bio. Sci., 2015, 5, 25-43.

12. J.K. Patra and K. Baek, J. of Nanomaterials, 2015, doi:org/10.1155/2014/417305.

13. M. Shah, D. Fawcett, S. Sharma, S.K. Tripathy and G.E. Jai poinern, Materials, 2015, 8, 72787308, doi:10.3390/ma8115377.

14. N. Bhuvana, and S. Vanitha, IOSR Journal of Applied Chemistry, 2016, 9, 49-53, ISSN:22785736.

15. A. Umer, S. Naveed, N. Ramzan, R.M. Shahid, and M. Imran, REVISTA MATERIA, 2014, 19(3), 197-203, ISSN:1517-7076artigo11547.

16. K. Saranyaadevi, V. Subha, E.R.S. Ravindran and S. Renganthan, Int. J. of Chem. Tech. Research, 2014, 6(10), 4533-4541.

17. A.T. Joseph, P. Prakash and S.S. Narvi, Int. J. of Sci. Engi. and Techno., 2016, 4, 463-472, ISSN:2348-4098.

18. L. Rastogi and J. Arunachalam, Advanced materials letters, 2011, 4(7), 548-555.

19. S.A. Khan, M. Jameel, S. Kanwal and S. Shahid, Int. J. of Pharmaceut. Sci. and Research, 2017, 2(3), 29-39, ISSN: 2455-4685.

20. N. Pantidos, and L.E. Horsfall, J.of Nano medicine and technology, 2014, 5, doi:10.4712/21577439.1000233.

21. A.M. Ranjitham, G.S. Ranjani and G. Caroling, Int. J. of Pharm. Tech. Res., 2015, 8, 750769, ISSN: 0974-4304.

22. M.S. Usman, N.A. Ibrahim, K. Shameli, N. Zainduddi, and W.M Yunus, Molecules, 2012, 17, 1429-1436. 